\title{
New horizons in breast cancer: the promise of immunotherapy
}

\author{
L. de la Cruz-Merino ${ }^{1}$ (D) N. Palazón-Carrión ${ }^{1} \cdot$ F. Henao-Carrasco ${ }^{1} \cdot$ E. Nogales-Fernández ${ }^{1} \cdot$ M. Álamo-de la Gala ${ }^{1}$. \\ A. Vallejo-Benítez ${ }^{2} \cdot$ M. Chiesa ${ }^{3} \cdot$ V. Sánchez-Margalet ${ }^{4}$ on behalf of GEICAM (Spanish Breast Cancer Research Group) \\ and GÉTICA (Spanish Group for Cancer Immuno-Biotherapy)
}

Received: 28 April 2018 / Accepted: 4 June 2018 / Published online: 18 June 2018

(c) The Author(s) 2018

\begin{abstract}
Immunology and immunotherapy of cancer is an expanding field in oncology, with recent great achievements obtained through the new successful approaches implemented to circumvent immune evasion, which is undoubtedly considered a novel hallmark of cancer. Translational research in this topic has revealed targets that can be modulated in the clinical setting with new compounds and strategies. Like most of the tumors, breast cancer is considered a complex and heterogeneous disease in which host immune responses have been also recently demonstrated of critical relevance. T infiltrating lymphocyte measurement is suggested as a powerful new tool necessary to predict early breast cancer evolution, especially for the her2-positive and triple-negative subtypes. Other biomarkers in tissue and peripheral blood are under intense scrutiny to ascertain their eventual role as prognostic and/or predictive factors. This background has fueled the interest in developing clinical research strategies to test activity of modern immunotherapy in breast cancer, which constitutes the main focus of this review.
\end{abstract}

Keywords Breast $\cdot$ Cancer $\cdot$ Immunology $\cdot$ Immunotherapy $\cdot$ Clinical $\cdot$ Trials

\section{Introduction}

It is well recognized that breast cancer (BC) is such a heterogeneous disease with different molecular subtypes that translate into different biological behaviors and aggressiveness, explaining the diverse clinical evolutions observed in patients. Beyond classification into different histologic and molecular subtypes (luminal A/B, her2 and triple negative), in the last few years, new diagnostic approaches have enriched and improved our knowledge of this disease. In this sense, study of the BC microenvironment, especially with respect

L. de la Cruz-Merino

lucme12@yahoo.es

1 Clinical Oncology Department, Hospital Universitario Virgen Macarena, Avda Dr Fedriani s/n, 41009 Seville, Spain

2 Pathology Department, Hospital Universitario Virgen Macarena, Avda Dr Fedriani s/n, 41009 Seville, Spain

3 Spanish Breast Cancer Research Group (GEICAM), 28703 Madrid, Spain

4 Department of Medical Biochemistry and Molecular Biology and Immunology, Medical School, Hospital Universitario Virgen Macarena, Avda Dr Fedriani s/n, 41009 Seville, Spain to its immune components, may proportionate relevant prognostic and even predictive information that could eventually guide therapeutic decisions. Moreover, in the era of modern immunotherapy with immune checkpoint blockers (ICB), a plethora of clinical trials have been conducted or planned and first data have already been communicated, showing some degree of activity, especially in certain subpopulations. In this work, we try to summarize an updated review of the ongoing clinical research with immunotherapy in BC.

\section{Rationale for immunotherapy in BC: TILs and PD-L1}

Cancer immune edition has been revealed as a major hallmark of cancer. As tumors evolve, their specific stroma turns into a microenvironment with an overwhelming density of immunosuppressive cells such as regulatory T cells (Treg), tumor-associated macrophages (TAMs), myeloid-derived suppressor cells (MDSC) and T cells of Th-2 type response, among others $[1,2]$.

In $\mathrm{BC}, \mathrm{T}$ infiltrating lymphocytes (TILs) as a whole (helper $\mathrm{T}$ cells, cytotoxic $\mathrm{T}$ cells, Treg) nowadays represent a putative prognostic and predictive biomarker $[3,4]$. 
Recently, the International TIL Working Group proposed a standardized methodology to measure TILs in BC through visual assessment on hematoxylin and eosin sections [5]. Main conclusions of this consensus were that full sections are preferred over biopsies (although core biopsies were acceptable in the neoadjuvant setting), and TILs should be measured as a continuous variable as a percentage. Besides, stromal compartment is at the moment the location where TILs may proportionate more valuable information from a clinical point of view. Although there is no formal recommendation for a clinical relevant threshold yet, the TIL Working Group describes the term lymphocyte predominant breast cancer (LPBC) for tumors that harbor a very high immune infiltrate ( $>50-60 \%$ of TILs). These LPBC tumors may represent from 13 to $30 \%$ of $\mathrm{BC}$ overall and there are differences depending on the specific subtypes, being more frequent in triple-negative breast cancer (TNBC) and her2+ subpopulations [6].

Association between pathologic complete response (pCR) upon neoadjuvant treatment and presence of TILs has been described in several studies [7-9]. This is quite relevant in daily practice as it may represent a new robust and reproducible predictive factor. One of the latest works in this scenario was conducted by the German Breast Cancer Group (GBG) which studied 3771 patients treated with different neoadjuvant chemotherapy schedules in the context of the GeparDuo, Gepartrio, GeparQuattro, GeparQuinto, GeparSixto and GeparSepto trials [6]. BC specimens were quantified on $\mathrm{H} \& \mathrm{E}$ sections of core biopsies following the TIL Working Group guidelines. All BC molecular subtypes were represented, her2+, luminal B her2- and TNBC (38, 37 and 25\%, respectively), and three predetermined subgroups with respect to TILs density were considered: high TILs-LPBC (60-100\%), intermediate TILs (11-59\%) and low TILs (0-10\%). The so-called LPBC cases were more frequent in the TNBC and her $2+$ subtypes (30 and 19\%, respectively). Increased concentration of TILs was a predictor of response, since pCR was achieved in $20 \%$ of tumors with low TILs, $27 \%$ of tumors with intermediate TILs, and $44 \%$ of tumors with high TILs, with statistically significant differences ( $p$ 0.0001) and confirming the findings of previous studies. This GBG project also evaluated TILs as a prognostic factor, and disease-free survival (DFS) and overall survival (OS) of 2570 patients were retrieved. With a median follow-up for OS and DFS of 62.8 months and 63.3 months, respectively, increased TIL concentration assessed as a continuous variable was related to longer DFS and OS in TNBC and longer DFS but with no impact in OS in her2+ patients. In the case of luminal B her2- patients, TIL concentration was not associated with DFS, and, surprisingly, a low TIL concentration was significantly associated with longer OS than was a high TIL concentration, showing opposite results with respect to the TNBC and her2+ populations [6]. Explanations to these results are yet to be elucidated.
TIL value as a prognostic factor has been assessed in multiple other studies although most of them are retrospective in nature [10-12]. Through these works, it can be inferred that stromal TILs represent a reliable prognostic factor in TNBC for DFS and OS. The above may also hold true for her $2+$ patients, although data are less robust. Interestingly, when TILs are dissected into their different subtypes and CD8+ T cells are specifically analyzed, a higher density of these cytotoxic cells in the stroma correlates with better long-term BC-specific survival in the TNBC population. In the case of FOXP3+ Tregs, these immunosuppressive cells hinder an effective activity of cytotoxic $\mathrm{T}$ cells, and thus the CD8+ T cells/FOXP3+ Tregs ratio could be another interesting parameter to be evaluated in this context $[13,14]$. Some studies have revealed that a high scoring in the CD8+ $\mathrm{T}$ cells/FOXP3+ Tregs ratio predicts long-term relapse-free survival and OS and, therefore, it could represent another valuable prognostic tool in the coming future $[13,14]$.

In addition to TILs and their subtypes, other immune elements have been recently evaluated in BC. This is the case of the programmed death-ligand 1 (PD-L1), one of the two ligands described for the co-inhibitory molecule PD-1 which can be expressed on tumor and immune cells. The PD1/PD-L1 axis is a major target for immunotherapy, and its modulation seems to be one of the main determinants for efficacy with the new immunomodulatory monoclonal antibodies [15, 16]. A recent study in $110 \mathrm{BC}$ patients demonstrated PD-L1 positivity (>1\%) in $23.6 \%$ of the patients, being more frequent in the TNBC, luminal B and her2+ populations (42.9, 41.7 and $29.4 \%$, respectively) with respect to luminal A patients (9.3\% of positivity to PD-L1) [17].

$\mathrm{BC}$ is considered a "cold" or non-immunogenic tumor. However, as previously mentioned, this is a quite heterogeneous disease in which TILs and PD-L1 (as surrogates of basal immunogenicity) can vary enormously from tumor to tumor. The great success achieved with immune checkpoint antibodies in different tumors, especially targeting inhibitory molecules, has also opened the door to a great research effort with immunotherapy in BC. Most of the clinical trials initiated have tried to enrich populations limiting recruitment to specific subtypes (mainly TNBC) and/or PD-L1 positivity. In the following sections, we will describe the first clinical results obtained with these strategies in $\mathrm{BC}$, and the trials currently ongoing that will shed light on the eventual role of immunotherapy in this disease.

\section{First results with immunomodulatory agents as single therapy}

Monoclonal antibodies (MAbs) against immune checkpoint inhibitors have demonstrated clinical activity in several cancer types as single therapy. Therefore, also in 
$\mathrm{BC}$, a set of different clinical trials has been initiated for testing the efficacy and safety of these new compounds. In this section, we will try to analyze the main characteristics of them and the first results already reported (Table 1).

\section{A phase IB multicohort study of pembrolizumab in subjects with advanced solid tumors (Keynote-012) (NCT01848834)}

Keynote-012 study was a phase Ib trial designed to evaluate the safety and activity of the anti-PD-1 monoclonal antibody pembrolizumab [18] in a set of PD-L1-positive advanced tumors that included a cohort of TNBC patients. Although most of the 32 TNBC patients included were heavily pretreated $[19,20]$, treatment was deemed safe with low-grade 3-4 toxicities. Sixteen (62\%) patients experienced a treatment-related AE of any grade, including two $(8 \%)$ with one or more events of grade 3 . The most common treatment-related AEs of any grade included arthralgia (18.8\%), fatigue (18.8\%), myalgia (18.8\%), and nausea (15.6\%). Overall response rate (ORR) observed was $18.5 \%$, and median progression free survival (PFS) and overall survival (OS) were 1.9 months and 10.2 months, respectively. The disease control rate (DCR) that included stabilization of disease (SD) was $25.9 \%$ and three of the five responders were considered to have long-lasting benefit from pembrolizumab.

To summarize, pembrolizumab may provide long-lasting responses in patients with $\mathrm{mTNBC}$, with $22 \%$ of patients alive at 2 years in this study, thereby supporting its further development for heavily pretreated patients who have generally poor prognosis [20]. Keynote-012 is one of the first studies to offer proof of principle of activity for anti-PD1
MAb as single therapy in populations of TNBC patients heavily pretreated.

\section{Phase IB study of pembrolizumab in subjects with select advanced solid tumors (Keynote-028) (NCT02054806)}

A second phase 1 study that has communicated data is the Keynote- 028 trial. This was a multicohort, open-label phase $1 \mathrm{~b}$ study evaluating the safety and efficacy of pembrolizumab in patients with PD-L1-positive advanced solid tumors, including BC [21]. Twenty-five patients with ER +/ her2-negative BC and PD-L1-positivity were enrolled. Median age was 53 years (range 36-79). 76\% of the BC patients have received $\geq 3$ lines of therapy for advanced disease, including $44 \%$ who received $\geq 5$ prior lines. After a median follow-up duration of 7.3 months, ORR was $14.0 \%$ and CBR was $23.0 \%$. The three patients who responded to treatment had remained on study for $\geq 26$ weeks at time of data cutoff. Median time to response was 8 weeks and, again, median DOR was not reached (range 8.7-44.3 weeks).

With respect to toxicity, serious AEs were reported in $16 \%$ of the cases and main irAEs observed were autoimmune hepatitis, hyperthyroidism, hypothyroidism, and pneumonitis. No treatment-related discontinuations or deaths occurred.

The two aforementioned studies included patients from two different BC subtypes (TNBC and ER +/her2-) although both studies shared as a prerequisite that tumors should be PD-L1+. Positivity for PD-L1 and TIL infiltration seems higher in TNBC subtypes. Whether these findings may translate into an improvement in clinical outcomes with anti-PD1 MAbs is a question yet to be resolved.

Table 1 First clinical results with monoclonal antibodies against immune checkpoint inhibitors in breast cancer as single therapy

\begin{tabular}{|c|c|c|c|c|c|c|c|c|}
\hline Trial (reference) & Phase & $n$ & BC subtype & Median age & Immunotherapy & $\begin{array}{l}\text { PDL1 status } \\
(\% \text { pos })\end{array}$ & ORR (\%) & $\begin{array}{l}\text { Grade } 3-4 \\
\text { toxicity } \\
(\%)\end{array}$ \\
\hline $\begin{array}{l}\text { Keynote } 012 \text { (NCT01848834) } \\
\text { [18] }\end{array}$ & IB & 32 & TNBC & 50.5 & Pembrolizumab & 58.6 & 18.5 & 15.6 \\
\hline $\begin{array}{l}\text { Keynote-028 (NCT02054806) } \\
{[21]}\end{array}$ & IB & 25 & $\begin{array}{l}\text { ER+ } \\
\text { HER2- }\end{array}$ & 53 & Pembrolizumab & 19 & 14 & 16 \\
\hline $\begin{array}{l}\text { Keynote-086 (NCT02447003) } \\
{[22]}\end{array}$ & II & 170 & TNBC & 54 & Pembrolizumab & 62 & 4.7 & 12 \\
\hline $\begin{array}{l}\text { Keynote-086 (NCT02447003) } \\
{[23]}\end{array}$ & II & 52 & TNBC & 53 & Pembrolizumab & 100 & 23.1 & 8 \\
\hline $\begin{array}{l}\text { PCD4989 g trial (NCT01375842) } \\
\text { [25] }\end{array}$ & IA & 112 & TNBC & 48 & Atezolizumab & 74 & 10 & 11 \\
\hline $\begin{array}{l}\text { JAVELIN (NCT01943461) } \\
\text { [28] }\end{array}$ & IA & 168 & Mixed & $\mathrm{N} / \mathrm{R}$ & Avelumab & 63.2 & 4.8 & 13.7 \\
\hline
\end{tabular}

$B C$ breast cancer, $E R$ estrogen receptor, $O R R$ overall response rate, pos positivity, $T N B C$ triple-negative breast cancer, $n$ sample size, $N / R$ not registered 


\section{Phase 2 study of pembrolizumab monotherapy for previously treated metastatic triple-negative breast cancer (mTNBC): cohort A Keynote-086 (NCT02447003)}

The use of pembrolizumab as monotherapy in metastatic TNBC was further evaluated in a phase II, single-arm multicohort study (Keynote-086). Patients were divided into three cohorts depending on their clinical treatment setting and PD-L1 status: cohort A (previously treated patients and PD-L1 unselected), cohort B (no prior systemic anticancer therapy and a tumor PD-L1 combined positive score $[\mathrm{CPS}] \geq 1 \%$ ) and cohort C. CPS evaluates PD-L1 expression on tumor and immune cells. Early data from cohort A [22] and B [23] have been reported, not for cohort C.

\section{Keynote- 086 cohort A results}

A total of 170 patients were enrolled in cohort A Keynote-086 [22] which examined the efficacy and safety of pembrolizumab in previously treated patients with $\geq 1$ prior chemotherapy for metastatic disease $\mathrm{mTNBC}$, regardless of PD-L1 expression. Of 170 patients enrolled with a median age of 54 years, $44 \%$ had received $\geq 3$ prior lines of therapy, and $74 \%$ had visceral metastasis. $62 \%$ of the patients had PD-L1+tumors (CPS $\geq 1 \%$ ). After a median follow-up of 10.9 months, 9 (5\%) patients remained on pembrolizumab. Treatment-related adverse events of any grade and grade 3-4 occurred in 60 and $12 \%$ of patients, respectively; $4 \%$ discontinued due to AEs. ORR was $4.7 \%$, and this was independent of PD-L1 expression although the unique complete responder had PD-L1-positive disease. One patient had a complete response to pembrolizumab monotherapy, seven patients (27\%) had a reduction in target lesions (PRs) and 35 patients had SD. The median duration of response and PFS was 6.3 months (range $1.2+$ to $10.3+$ ) and 2.0 months $(95 \% \mathrm{CI}$ 1.9-2.0), respectively. Median overall survival was 8.9 months and at 9 months, $100 \%$ of responders were alive, as were $89.6 \%$ of stable patients, compared with $39.0 \%$ of patients failing to show a benefit. In subgroup analyses, ORR was improved in patients with a low tumor burden, normal LDH at baseline and non-visceral disease and it appeared regardless of PD-L1 expression.

\section{Keynote- 086 cohort $B$ results}

This cohort of the Keynote-086 trial [23] assessed the safety and antitumor activity of pembrolizumab as first-line therapy for patients with PD-L1-positive mTNBC. 79 of the first 137 patients included with PD-L1-evaluable tumors (58\%) had PD-L1 CPS $\geq 1 \%$. Of the first 52 patients enrolled, median age was $53,40 \%$ had elevated $\mathrm{LDH}, 69 \%$ had visceral metastases, and $87 \%$ received prior (neo)adjuvant therapy.
After a median follow-up of 7.0 months (range 4.4-12.5), $15(29 \%)$ patients remained on pembrolizumab. Treatmentrelated AEs occurred in 37 (71\%) patients, most commonly fatigue $(31 \%)$, nausea $(15 \%)$, and diarrhea $(13 \%)$. Four $(8 \%)$ patients experienced five grade 3-4 treatment-related AEs. No patients died or discontinued pembrolizumab due to an AE. ORR was 23\% (95\% CI 14-36\%) and best overall response was CR in 4\%, PR in 19\%, SD in $17 \%$ and $\mathrm{PD}$ in $58 \%$ of the patients. Median duration of response was 8.4 months, with eight $(67 \%)$ responses ongoing at cutoff in Nov 2016. Median PFS was 2.1 months and the estimated 6-month PFS rate was 29\%. These data from the first 52 patients enrolled in Keynote-086 cohort B suggest that pembrolizumab monotherapy has a manageable safety profile and promising antitumor activity as first-line therapy for PDL1-positive mTNBC. Tumor shrinkage was more common in cohort B than in the previously treated cohort A [22] with any degree of PD-L1 status.

Beyond PD-L1, relationship between the ORR and stromal TIL levels was analyzed in the cohorts A and B of the Keynote-086 trial. At this point, the ORR with pembrolizumab in patients with stromal TIL level above or below the median was, respectively, 6.4 versus $1.9 \%$ in cohort A and 39.1 versus $8.7 \%$ in cohort B. In addition, taking into account the combined cohorts, higher stromal TIL levels were associated with significantly improved ORR as a continuous variable. Therefore, as described in this substudy of the Keynote-086 trial, pembrolizumab response showed a significant relationship to stromal TIL levels [24].

\section{A Phase I, open-label, dose-escalation study of the safety and pharmacokinetics of atezolizumab administered intravenously as a single agent to patients with locally advanced or metastatic solid tumors or hematologic malignancies (PCD4989 g trial) (NCT01375842)}

The PCD4989 $\mathrm{g}$ trial studied the safety and activity of atezolizumab in TNBC $[25,26]$. This phase 1a trial had a dose escalation and an expansion cohort. The updated long-term clinical outcomes and biomarker analyses of a cohort of 115 mTNBC patients from this study were reposted by Schmid and Colleagues at the AACR annual meeting 2017 [27]. Of the 112 patients evaluable for response, 93 had received at least two lines of prior therapy. Two-third of tumors had high levels of PD-L1 expression, defined as $\geq 5 \%$ positive immuneinfiltrating cells. The ORR was $10 \%$ overall and $13 \%$ in the group with PD-L1 positivity. ORR was $26 \%$ in patients who were treated in the first-line setting and $11 \%$ for the secondline group. Disease control (including stabilization of disease) was achieved in $30 \%$ of patients overall and $58 \%$ of those treated in the first line. Among responders, median duration of response was 21.1 months. Median OS was 9.3 months 
in all patients. The 1-year overall survival rate was $41 \%$ and the 2-year survival rate was $22 \%$. Among responders, 1 - and 2-year survival rates were 100 vs 33 and $11 \%$, respectively, for nonresponders. Exploratory analysis evaluated biomarkers and found that higher response rates seemed to be associated with higher levels of tumor-infiltrating lymphocytes $(>10 \%$ TIL), CD8+ T cells ( $>1.35 \%$ intratumoral CD8+TIL), and PD-L1 expression (but patients with lower PD-L1 expression also benefited from treatment with atezolizumab). Only $11 \%$ of patients experienced treatment-related grade $3-4$ side effects, which led to treatment discontinuation in only in the $3 \%$ of the cases.

\section{Avelumab for metastatic or locally advanced previously treated solid tumors (JAVELIN Solid Tumor): a phase 1a, multicohort, dose-escalation trial (NCT01943461)}

The fully human anti-PD-L1, IgG1 antibody avelumab was assessed as a single agent in 168 patients with unselected advanced or metastatic BC in the open-label, dose-escalation JAVELIN phase I trial [28]. The BC subtype distribution included approximately $43 \% \mathrm{ER}+/ \mathrm{PR}+/ \mathrm{her} 2-, 35 \%$ TNBC, and $16 \%$ her2+ tumors. The ORR in this unselected population was quite modest (4.8\%) with subgroup analyses demonstrating a higher RR in patients with TNBC (ORR $8.6 \%$ ). In this study, PD-L1 expression was not related to the efficacy of the treatment. With respect to toxicity, avelumab demonstrated an acceptable safety profile with few cases $(17 \%)$ of grade 3-4 events: autoimmune disorder (5.6\%), increased blood creatine phosphokinase (3.7\%), and increased aspartate aminotransferase (3.7\%).

\section{First results in clinical trials with MAbs against immune checkpoints inhibitors in the context of combinations}

The favorable safety profile of the immune checkpoint blockers (ICB) and the eventual synergism that could be elicited with other active therapies against $\mathrm{BC}$ gave pace to numerous studies exploring different combinations. In the following lines, we revise some initial results reported of clinical trials with this approach in mind.

\section{A phase Ib study of the safety and pharmacology of atezolizumab administered with bevacizumab and/or with chemotherapy (nab-paclitaxel) in patients with advanced solid tumors (NCT01633970)}

The MAb anti-VEGF bevacizumab has shown to inhibit the infiltration of immune-suppressive cells such as Tregs and
MDSCs in murine models. Therefore, a phase 1b open-label, multicenter study evaluated atezolizumab in combination with bevacizumab and/or chemotherapy in patients with locally advanced or metastatic solid tumors [29]. One of the arms ( $\mathrm{F}$ arm) of this multicohort study included patients with locally advanced or metastatic TNBC previously treated with $\leq 2 \mathrm{w}$ systemic cytotoxic therapies. Patients assigned to this $\mathrm{F}$ arm received atezolizumab $800 \mathrm{mg}$ IV every 2 weeks followed by nab-paclitaxel $125 \mathrm{mg} / \mathrm{m}^{2}$ IV every week for at least four cycles in the absence of PD or unacceptable toxicity. Patients received a median of five prior systemic treatments. In this trial, the confirmed ORR was $41.7 \%$. Eleven out of 17 responses were ongoing at time of data cutoff. ORR for patients who received atezolizumab plus nab-paclitaxel schedule as first- and second-line therapy was 66.7 and $25.0 \%$, respectively. All-grade treatment-related AEs were observed in $100 \%$ of patients with grade 3-4 AEs occurring in $56 \%$ of patients. Treatment-related grade 3-4 AEs observed in $\geq 5 \%$ of patients included neutropenia ( $41 \%)$, thrombocytopenia (9\%), and anemia (6\%). Five patients had to abandon chemotherapy with nab-paclitaxel due to grade 2 fatigue and peripheral neuropathy.

\section{A phase Ib/Il trial evaluating the efficacy of pembrolizumab (MK-3475) and trastuzumab in patients with trastuzumab-resistant, her2-positive metastatic breast cancers: PANACEA (IBCSG 45-13/BIG 4-13/Keynote-014) (NCT02129556)}

Preclinical data suggest that anti-PD1 therapy may restore $\mathrm{T}$ cell cytotoxicity reverting trastuzumab resistance [30]. Keynote-014 is an international phase Ib/II trial [31] testing trastuzumab in combination with pembrolizumab in patients with trastuzumab-resistant her2-positive $\mathrm{mBC}$. The phase $\mathrm{Ib}$ portion of this trial used a standard $3+3$ design to determine the recommended phase 2 dose (RP2D) of standard-dose trastuzumab with three pembrolizumab dose levels. In the phase 2 portion of the study, pembrolizumab at the RP2D (200 mg Q3 W) was given with trastuzumab $(6 \mathrm{mg} / \mathrm{kg}$ iv. every 3 weeks) until disease progression or lack of tolerability. Tumors were assessed centrally for her2 positivity, PD-L1 status and for quantity of tumor-infiltrating lymphocytes (TILs).

146 patients were screened to enroll 58 patients. Of screened patients, $52 \%$ were PD-L1 positive. Median TILs of patients in the PD-L1 + cohort were 2 and $0 \%$ in the PD-L1 - cohort. Of the enrolled patients, median age was 51 years, $69 \%$ had visceral metastases, $72 \%$ had received prior T-DM1, 38\% of patients were ER positive and 62\% were ER negative. PD-L1 positivity rates were also not significantly different by ER status ( $p=0.5$ ). In the PD-L1-positive intent-to-treat population, the ORR was $15 \%$ and disease control rate was $25 \%$. In a subgroup of PD-L1-positive patients with $5 \%$ or more TILs in the metastatic lesion, the 
ORR rose up to $39 \%$ and the disease control rate reached $47 \%$ suggesting that, at least in this setting, quantification of TILs may help to identify patients who will most benefit most from this treatment. No responses were observed in the PD-L1-negative cohort. With respect to safety, pembrolizumab with trastuzumab was well tolerated, with grade 1-2 fatigue as the most commonly reported adverse event (21\%). The most common irAEs reported were hyper- and hypothyroidism $(6.7 \%$ grade $1-2)$ and pneumonitis $(3.4 \%$ grade 3-4).

\section{An open-label, single-arm multicenter phase $1 \mathrm{~b} / 2$ study to evaluate the efficacy and safety of eribulin mesylate in combination with pembrolizumab in subjects with metastatic triple-negative breast cancer (mTNBC) (ENHANCE-1/KEYNOTE-150; Study 218) (NCT02513472)}

This open-label phase $1 \mathrm{~b} / 2$ trial enrolled patients with mTNBC treated with $\leq 2$ prior lines of CT for metastatic disease. Tolaney and Colleagues [32] reported data from 82 of 107 enrolled patients (data cut-off Nov 1, 2016). Phase $1 \mathrm{~b}$ included a safety cohort of $\geq 6$ patients who received IV eribulin $1.4 \mathrm{mg} / \mathrm{m}^{2}$ on day 1 and 8 and IV pembrolizumab $200 \mathrm{mg}$ on day 1 of a 21-day cycle to evaluate safety and tolerability. In Phase 2, patients were enrolled based on prior chemotherapy in the metastatic setting [0 vs 1-2 lines] to evaluate ORR. The combination of eribulin and pembrolizumab resulted in an ORR of $26.4 \%$ (95\% CI, 18.3-35.9). Three complete responses were observed; one of which was in a patient with a PD-L1-negative tumor.

In the evaluable analysis set $(n=106)$, patients who were PD-L1 positive had an ORR of $30.6 \%$ and patients who were PD-L1 negative had an ORR of $22.4 \%$. Patients with $\mathrm{mTNBC}$ who had no prior chemotherapy treatment in the metastatic setting $(n=65)$ had an ORR of $29.2 \%$ (95\% CI 18.6-41.8) and patients who received one or two prior lines of chemotherapy $(n=41)$ had an ORR of $22.0 \%$ (95\% CI 10.6-37.6). The median duration of response was 8.3 months. The clinical benefit rate (CBR) was $36.8 \%$. Median OS and median PFS for all patients in the trial ( $n=107)$ were 17.7 months (95\% CI 13.7-not estimable) and 4.2 months (95\% CI 4.1-5.6), respectively. Treatmentemergent adverse events (TEAEs) for the combination regimen were comparable to those observed with each treatment as a monotherapy. Most common AEs were fatigue (73.2\%), nausea $(51.2 \%)$, peripheral sensory neuropathy (46.3\%), alopecia $(43.9 \%)$, and pyrexia (36.6\%). Most common grade 3-4 AEs related to eribulin were neutropenia $(29.3 \%)$, peripheral neuropathy $(8.5 \%)$, and asthenia/fatigue $(7.3 \%)$. Grade 3-4 immune-related AEs related to pembrolizumab were observed in $19.5 \%$ of patients. Three patients had grade
5 events (respiratory failure, pleural effusion, and multiple organ failure; none of them related to the study drug).

To sum up, the combination of eribulin and pembrolizumab was well tolerated and demonstrated activity in patients with mTNBC regardless of PD-L1 status or prior treatment with chemotherapy. The combination resulted in improved outcomes and comparable TEAEs to those observed in monotherapy.

At this moment, dozens of clinical trials with many different combinatorial strategies (chemotherapy, radiotherapy, vaccines, hormone therapy, etc.) are running and will release results in the coming years.

\section{Phase III trials with immune checkpoint blockers in BC}

Although clinical research with ICB in BC is taking the first steps and many uncertainties are yet to be answered, there are already phase III clinical trials running which may proportionate critical information to elucidate the role of this strategy in BC, especially in the TNBC population.

In mTNBC, many clinical trials are currently ongoing (Table 2). The Keynote-119 trial randomize pretreated mTNBC patients to receive either single-agent pembrolizumab $200 \mathrm{mg}$ every 3 weeks for up to 35 administrations or single-agent chemotherapy chosen by the treating physician, consisting of either capecitabine, eribulin, gemcitabine, or vinorelbine [33]. Main objective is that pembrolizumab extends PFS and/or OS compared to control therapy.

Most of the phase 3 trials in mTNBC are focused on the first-line setting. In this context, the Keynote-355 [34] compares safety and efficacy of pembrolizumab plus chemotherapy (three options, weekly paclitaxel, weekly nab-paclitaxel or carboplatin-gemcitabine) versus placebo. The Impassion130 [35] study with atezolizumab tries to determine the efficacy and safety of atezolizumab or placebo in combination with nab-paclitaxel in mTNBC patients. The Impassion 131 trial [36] shares a design quite similar to the previous and tries to evaluate the efficacy and safety of atezolizumab administered in combination with paclitaxel compared with placebo in combination with paclitaxel in patients with previously untreated, inoperable locally advanced or mTNBC, centrally confirmed. Last but not least, the Impassion 132 [37] completes this set of phase 3 trials with atezolizumab but using as chemotherapeutic schedule the doublet carboplatin-gemcitabine in patients with early relapsing recurrent (inoperable locally advanced or metastatic) TNBC.

Undoubtedly, the aforementioned trials will clarify the role of modern immunotherapy in mTNBC, and it is easy to speculate that these results, when available, will 
Table 2 Ongoing phase 3 clinical trials with immune checkpoint inhibitors in BC

\begin{tabular}{|c|c|c|c|c|}
\hline Trial (reference) & $n$ & Experimental arm & Control arm & Clinical setting \\
\hline Keynote 119 (NCT02555657) [33] & 640 & Pembrolizumab & $\begin{array}{l}\text { CT (capecitabine, eribulin, gemcit- } \\
\text { abine, vinorelbine) }\end{array}$ & mTNBC pretreated \\
\hline Keynote-355 (NCT02819518) [34] & 858 & $\begin{array}{l}\text { Pembrolizumab + CT (paclitaxel, } \\
\text { nab-paclitaxel, carboplatin+ } \\
\text { gemcitabine) }\end{array}$ & $\begin{array}{l}\mathrm{CT} \text { (paclitaxel, nab-paclitaxel, carbo- } \\
\text { platin + gemcitabine) }\end{array}$ & mTNBC 1st line \\
\hline IMpassion131 (NCT03125902) [36] & 540 & Atezolizumab + paclitaxel & Paclitaxel & mTNBC 1st line \\
\hline IMpassion130 (NCT02425891) [35] & 285 & Atezolizumab + nab-paclitaxel & Nab-paclitaxel & mTNBC 1st line \\
\hline IMpassion132 (NCT03371017) [37] & 350 & $\begin{array}{l}\text { Atezolizumab }+ \\
\text { gemcitabine }+ \\
\text { carboplatin or capecitabine }\end{array}$ & $\begin{array}{l}\text { Gemcitabine + carboplatin or capecit- } \\
\text { abine }\end{array}$ & mTNBC 1st line \\
\hline Keynote-522 (NCT03036488) [38] & 855 & $\begin{array}{l}\text { Pembrolizumab }+ \text { paclitaxel }+ \text { carbo- } \\
\text { platin } \times 4 \text { cycles followed by AC or } \\
\text { EC } \times 4 \text { cycles }\end{array}$ & $\begin{array}{r}\text { Paclitaxel }+ \text { Carboplatin } \times 4 \text { cycles } \\
\text { followed by AC or } \mathrm{EC} \times 4 \text { cycles }\end{array}$ & TNBC neoadjuvant \\
\hline NCT03281954 [39] & 1520 & $\begin{array}{l}\text { Atezolizumab }+ \text { paclitaxel }+ \text { carbopl- } \\
\text { atin } \times 4 \text { cycles followed by AC or } \\
\text { EC } \times 4 \text { cycles }\end{array}$ & $\begin{array}{r}\text { Paclitaxel }+ \text { Carboplatin } \times 4 \text { cycles } \\
\text { followed by AC or } \mathrm{EC} \times 4 \text { cycles }\end{array}$ & TNBC neoadjuvant \\
\hline IMpassion031 (NCT03197935) [40] & 204 & $\begin{array}{l}\text { Atezolizumab and nab-paclitaxel } \\
\text { followed by doxorubicin and cyclo- } \\
\text { phosphamide }\end{array}$ & $\begin{array}{l}\text { Nab-paclitaxel followed by doxoru- } \\
\text { bicin and cyclophosphamide }\end{array}$ & TNBC neoadjuvant \\
\hline $\begin{array}{l}\text { NeoTRIPaPDL1 (NCT02620280) } \\
\text { [41] }\end{array}$ & 272 & $\begin{array}{l}\text { Atezolizumab + carboplatin }+ \text { nab- } \\
\text { paclitaxel }\end{array}$ & Carboplatin + nab-paclitaxel & TNBC neoadjuvant \\
\hline
\end{tabular}

$T N B C$ triple-negative breast cancer, $n$ sample size

determine also the clinical development of ICB in the rest of $\mathrm{BC}$ subtypes.

In addition to the advanced disease, some phase III trials with ICB (especially pembrolizumab and atezolizumab) in TNBC are running in the neoadjuvant setting (Table 2), in these studies drugs like nab-paclitaxel and carboplatin are frequently preferred and the main objective is generally pCR [38-41]. Awaiting first data of the previous trials, early results of the randomized phase 2 I-SPY 2 trial evaluating the addition of pembrolizumab with different chemotherapeutic schedules (nab-paclitaxel followed by doxorubicin and cyclophosphamide in the cohort $\mathrm{A}$ or carboplatin and paclitaxel followed by doxorubicin and cyclophosphamide in cohort B) have shown that immunotherapy could triple the pathologic complete response rates in TNBC and luminal A/B patients in the neoadjuvant setting [42].

\section{Conclusions and future perspectives}

Immunotherapy of cancer has emerged as a revolutionary strategy in many cancer types. Whether this approach would play a role into the therapeutic armamentarium of BC is nowadays an open question. There are some biological evidences that relate strongly the presence or absence of certain elements of the host immune response with a differential evolution in patients with BC. High density of TILs in the microenvironment, particularly of the T CD8+ subtype, is considered at this moment a robust predictive and prognostic factor at least in TNBC and her2+ populations. Although less studied, expression of PD-L1 seems related to a worse prognosis, with more aggressive tumor characteristics, including higher proliferation and hormone receptor negativity. Design of many of the clinical trials currently ongoing in $\mathrm{BC}$ has taken into account these variables so that most of them have been enriched with PD-L1-positive tumors. This could be an interesting strategy especially when using ICB as single therapy, where responses with immunomodulatory agents in BC are generally low. In this sense, in unselected populations, ORR with ICB is under $10 \%$ while in enriched subgroups they increase significantly (up to $39 \%$ in mTNBC, PD-L1+ and high TILs). Less clear is the validity of this approach in the context of combinations, since the rationale of the latter is the capacity of different antineoplastic therapies in turning the so-called "cold" tumors into "hot" or immunogenic ones. Preliminary results of some studies with ICB in BC suggest that PD-L1 positivity may not be as important as when ICB are used as single therapy. If this is the case, other more comprehensive approach would be advisable including the analysis of genomic platforms with the capacity of detecting immunogenicity, measure of mutational burden, identification of neoantigens with the ability to set off the danger signal to unleash immune responses, the gut microbiome and many others. Although immunotherapy in $\mathrm{BC}$ is raising great expectations, there remain 
many uncertainties that hopefully the aforementioned trials, with the inexcusable aid of translational research, will satisfactorily resolve.

\section{Compliance with ethical standards}

Conflict of interest Authors declare no conflict of interest with respect to this work.

Ethical approval Research involving human participants and/or animals: not applicable.

Informed consent Not applicable

Open Access This article is distributed under the terms of the Creative Commons Attribution 4.0 International License (http://creativeco mmons.org/licenses/by/4.0/), which permits unrestricted use, distribution, and reproduction in any medium, provided you give appropriate credit to the original author(s) and the source, provide a link to the Creative Commons license, and indicate if changes were made.

\section{References}

1. Schreiber RD, Old LJ, Smyth MJ. Cancer immunoediting: integrating immunity's roles in cancer suppression and promotion. Science. 2011;331(6024):1565-70. https://doi.org/10.1126/scien ce. 1203486 .

2. Swann JB, Smyth MJ. Immune surveillance of tumors. J Clin Investig. 2007;117(5):1137-46. https://doi.org/10.1172/jci31405.

3. Bohling SD, Allison KH. Immunosuppressive regulatory T cells are associated with aggressive breast cancer phenotypes: a potential therapeutic target. Mod Pathol. 2008;21(12):1527-32. https:// doi.org/10.1038/modpathol.2008.160.

4. Savas P, Salgado R, Denkert C, Sotiriou C, Darcy PK, Smyth MJ, et al. Clinical relevance of host immunity in breast cancer: from TILs to the clinic. Nat Rev Clin Oncol. 2016;13(4):228-41. https ://doi.org/10.1038/nrclinonc.2015.215.

5. Salgado R, Denkert C, Demaria S, Sirtaine N, Klauschen F, Pruneri G, et al. The evaluation of tumor-infiltrating lymphocytes (TILs) in breast cancer: recommendations by an International TILs working group 2014. Ann Oncol. 2015;26(2):259-71. https ://doi.org/10.1093/annonc/mdu450.

6. Denkert C, von Minckwitz G, Darb-Esfahani S, Lederer B, Heppner BI, Weber KE, et al. Tumour-infiltrating lymphocytes and prognosis in different subtypes of breast cancer: a pooled analysis of 3771 patients treated with neoadjuvant therapy. Lancet Oncol. 2018;19:40-50. https://doi.org/10.1016/S1470-2045(17)30904-X.

7. Denkert C, Loibl S, Noske A, Roller M, Muller BM, Komor M, et al. Tumor-associated lymphocytes as an independent predictor of response to neoadjuvant chemotherapy in breast cancer. J Clin Oncol. 2010;28(1):105-13.

8. Denkert C, von Minckwitz G, Brase JC, Sinn BV, Gade S, Kronenwett $\mathrm{R}$, et al. Tumor-infiltrating lymphocytes and response to neoadjuvant chemotherapy with or without carboplatin in human epidermal growth factor receptor 2-positive and triple-negative primary breast cancers. J Clin Oncol. 2015;33(9):983-91.

9. Issa-Nummer Y, Darb-Esfahani S, Loibl S, Kunz G, Nekljudova V, Schrader I, et al. Prospective validation of immunological infiltrate for prediction of response to neoadjuvant chemotherapy in her2-negative breast cancer-a substudy of the neoadjuvant GeparQuinto trial. PLoS One. 2013;8(12):e79775. https://doi. org/10.1371/journal.pone.0079775.
10. Dieci MV, Criscitiello C, Goubar A, Viale G, Conte P, Guarneri $\mathrm{V}$, et al. Prognostic value of tumor-infiltrating lymphocytes on residual disease after primary chemotherapy for triple-negative breast cancer: a retrospective multicenter study. Ann Oncol. 2014;25(3):611-8.

11. Dieci MV, Mathieu MC, Guarneri V, Conte P, Delaloge S, Andre F, et al. Prognostic and predictive value of tumor-infiltrating lymphocytes in two phase III randomized adjuvant breast cancer trials. Ann Oncol. 2015;26(8):1698-704.

12. Loi S, Michiels S, Salgado R, Sirtaine N, Jose V, Fumagalli D, et al. Tumor infiltrating lymphocytes are prognostic in triple negative breast cancer and predictive for trastuzumab benefit in early breast cancer: results from the FinHER trial. Ann Oncol. 2014;25(8):1544-50.

13. Ali HR, Provenzano E, Dawson SJ, Blows FM, Liu B, Shah M, et al. Association between CD8+ T-cell infiltration and breast cancer survival in 12,439 patients. Ann Oncol. 2014;25(8):1536-43. https://doi.org/10.1093/annonc/mdu191.

14. Ladoire S, Mignot G, Dabakuyo S, Arnould L, Apetoh L, Rébé C, et al. In situ immune response after neoadjuvant chemotherapy for breast cancer predicts survival. J Pathol. 2011;224(3):389-400. https://doi.org/10.1002/path.2866.

15. Cimino-Mathews A, Thompson E, Taube JM, Ye X, Lu Y, Meeker A, et al. PD-L1 (B7-H1) expression and the immune tumor microenvironment in primary and metastatic breast carcinomas. Hum Pathol. 2016;47:52-63. https://doi.org/10.1016/j.humpa th.2015.09.003.

16. Herbst RS, Soria JC, Kowanetz M, Fine GD, Hamid O, Gordon MS, et al. Predictive correlates of response to the anti-PD-L1 antibody MPDL3280A in cancer patients. Nature. 2014;515:563-7. https://doi.org/10.1038/nature14011.

17. Buisseret L, Garaud S, de Wind A, Van den Eynden G, Boisson A, Solinas C, et al. Tumor-infiltrating lymphocyte composition, organization and PD-1/PD-L1 expression are linked in breast cancer. OncoImmunology. 2017;6(1):e1257452. https://doi. org/10.1080/2162402X.2016.1257452.

18. Merck. Keytruda (pembrolizumab). In: Merck 2015. http://www. merck.com/product/usa/pi_circulars/k/keytruda/keytruda_pi.pdf. Accessed 9 April 2018.

19. Nanda R, Chow LQ, Dees EC, Berger R, Gupta S, Geva R, et al. Pembrolizumab in patients with advanced triple-negative breast cancer: phase Ib Keynote-012 study. J Clin Oncol. 2016;34(21):2460-7. https://doi.org/10.1200/jco.2015.64.8931.

20. Nanda R, Specht J, Dees EC. Pembrolizumab for metastatic triplenegative breast cancer (mTNBC): long-lasting responses in the phase Ib KEYNOTE-012 study. Eur Journ Cancer. 2017;243:S38.

21. Rugo HS, Delord JP, Im SA, Ott PA, Piha-Paul SA, Bedard PL, et al. Abstract S5-07: preliminary efficacy and safety of pembrolizumab (MK-3475) in patients with PD-L1-positive, estrogen receptor-positive $(\mathrm{ER}+) /$ her2-negative advanced breast cancer enrolled in Keynote-028. Cancer Res. 2016;76:S5-07. https:// doi.org/10.1158/1538-7445.sabcs15-s5-07.

22. Adams S, Schmid P, Rugo HS, Winer ER, Loirat D, Awada A, et al. Phase 2 study of pembrolizumab (pembro) monotherapy for previously treated metastatic triple-negative breast cancer (mTNBC): Keynote-086 cohort A. J Clin Oncol. 2017;35((15suppl)):abstr 1008.

23. Adams S, Loi S, Toppmeyer D, Cescon DW, De Laurentiis M, Nanda R, et al. Phase 2 study of pembrolizumab as first-line therapy for PD-L1-positive metastatic triple-negative breast cancer (mTNBC): Preliminary data from Keynote-086 cohort B. J Clin Oncol. 2017;35((15_suppl)):abstr 1088.

24. Loi S, Adams S, Schmid P, Cortes S, Cescon DW, Winer EP, et al. Relationship between tumor infiltrating lymphocyte (TIL) levels and response to pembrolizumab (pembro) in metastatic 
triple-negative breast cancer (mTNBC): results from Keynote-086. Annals of Oncology. 2017;28((5_suppl)):LBA13.

25. Chen DS, Irving BA, Hodi FS. Molecular pathways: next-generation immunotherapy-inhibiting programmed death-ligand 1 and programmed death-1. Clin Cancer Res. 2012;18(24):6580-7. https ://doi.org/10.1158/1078-0432.ccr-12-1362.

26. Emens LA, Braiteh FS, Cassier P, Delord J-P, Eder JP, Fasso $\mathrm{M}$, et al. Inhibition of PD-L1 by MPDL3280A leads to clinical activity in patients with metastatic triple-negative breast cancer (TNBC). Proceedings of the 106th Annual Meeting of the American Association for Cancer Research; 2015 Apr 18-22; Philadelphia (PA): AACR. Cancer Res. 2015;75(15_Suppl):abstr 2859. 10.1158/1538-7445.am2015-2859.

27. Schmid P, Cruz C, Braiteh FS. Atezolizumab in metastatic triplenegative breast cancer: long-term clinical outcomes and biomarker analyses. AACR Annual Meeting 2017; April 1-5, 2017; Washington DC. Abstract 2986.

28. Heery CR, O'Sullivan-Coyne G, Madan RA, Cordes L, Rajan A, Rauckhorst M, et al. Avelumab for metastatic or locally advanced previously treated solid tumours (JAVELIN solid tumor): a phase 1a, multicohort, dose-escalation trial. Lancet Oncol. 2017;18:58798. https://doi.org/10.1016/S1470-2045(17)30239-5.

29. Adams S, Robinson-Diamond J, Paige-Hamilton E, RaffinPohlmann P, Tolaney SM, Molinero L, et al. Phase Ib trial of atezolizumab in combination with nab-paclitaxel in patients with metastatic triple negative breast cancer (mTNBC). J Clin Oncol. 2016;34((15_Suppl.)):abstr 1009. https://doi.org/10.1200/ jco.2016.34.15_suppl.1009.

30. Müller P, Kreuzaler M, Khan T, Thommen DS, Martin K, Glatz $\mathrm{K}$, et al. Trastuzumab emtansine (T-DM1) renders her2+ breast cancer highly susceptible to CTLA-4/PD-1 blockade. Sci Transl Med. 2015;7(315):315ra188. https://doi.org/10.1126/scitranslm ed.aac4925.

31. Loi S, Andre F, Maibach R, Hui R, Bartsch R, Jerusalem G, et al. PANACEA (IBCSG 45-13/BIG 4-13): a phase Ib/II trial evaluating the efficacy of pembrolizumab and trastuzumab in patients with trastuzumab-resistant, her2-positive, metastatic breast cancer. Proceedings of the Thirty-Eighth Annual CTRC-AACR San Antonio Breast Cancer Symposium: 2015 Dec 8-12; San Antonio, TX. Philadelphia (PA): AACR; Cancer Res 2016;76 (4_Suppl), Abstr OT3-01-05.

32. Tolaney S, Savulsky C, Aktan G, et al. Phase $1 \mathrm{~b} / 2$ study to evaluate eribulin mesylate in combination with pembrolizumab in patients with metastatic triple-negative breast cancer. Cancer Res. 2016;76((4_Suppl)):5-15.

33. Winer EP, Dang T, Karantza V, Su SC. Keynote-119: a randomized phase III study of single-agent pembrolizumab (MK3475 ) vs single-agent chemotherapy per physician's choice for metastatic triple-negative breast cancer (mTNBC). J Clin Oncol. 2016;34((15_Suppl.)):abstr TPS1102. https://doi.org/10.1200/ jco.2016.34.15_suppl.tps1102.

34. Cortés J, Guo Z, Karantza V, Aktan G. KEYNOTE-355: Randomized, double-blind, phase III study of pembrolizumab (pembro) + chemotherapy (chemo) vs placebo $(\mathrm{PBO})+$ chemo for previously untreated, locally recurrent, inoperable or metastatic triple-negative breast cancer (mTNBC). J Clin Oncol. 2018;36((5_ Suppl.)):abstr TPS18. https://doi.org/10.1200/jco.2018.36.5_suppl .tps18.

35. Emens LA, Adams S, Loi S, Schneeweiss A, Rugo HS, Winer EP, et al. IMpassion130: a Phase III randomized trial of atezolizumab with nab-paclitaxel for first-line treatment of patients with metastatic triple-negative breast cancer (mTNBC). J Clin Oncol. 2016;34((15_Suppl.)):abstr TPS1104. https://doi.org/10.1200/ jco.2016.34.15_suppl.tps1104.

36. Hoffmann-La Roche. A Phase III, multicenter, randomised, double-blind, placebo-controlled study of atezolizumab (Anti-Pd-L1 Antibody) in combination with paclitaxel compared with placebo with paclitaxel for patients with previously untreated inoperable locally advanced or metastatic triple negative breast cancer. In: ClinicalTrials.gov. U.S. National Library of Medicine. 2017. https ://clinicaltrials.gov/ct2/show/NCT03125902. Accessed 10 April 2018.

37. Hoffmann-La Roche. A phase iii, randomised, double-blind, placebo-controlled, multicentre study of the efficacy and safety of atezolizumab plus chemotherapy for patients with early relapsing recurrent (inoperable locally advanced or metastatic) triplenegative breast cancer. In: ClinicalTrials.gov. U.S. National Library of Medicine. 2017. https://clinicaltrials.gov/ct2/show/ NCT03371017. Accessed 10 April 2018.

38. Merck Sharp \& Dohme Corp. A phase III, randomized, doubleblind study to evaluate pembrolizumab plus chemotherapy vs placebo plus chemotherapy as neoadjuvant therapy and pembrolizumab vs placebo as adjuvant therapy for triple negative breast cancer (TNBC). In: ClinicalTrials.gov. U.S. National Library of Medicine. 2017. https://clinicaltrials.gov/ct2/show/ NCT03036488. Accessed 10 April 2018.

39. NSABP Foundation Inc. A randomized, double-blind, phase III clinical trial of neoadjuvant chemotherapy with atezolizumab or placebo in patients with triple-negative breast cancer followed by adjuvant continuation of atezolizumab or placebo. In: ClinicalTrials.gov. U.S. National Library of Medicine. 2017. https://clinicaltr ials.gov/ct2/show/ NCT03281954. Accessed 10 April 2018.

40. Hoffmann-La Roche. A phase III randomized study to investigate the efficacy and safety of atezolizumab (Anti-PD-L1 Antibody) in combination with neoadjuvant anthracycline/nab-paclitaxelbased chemotherapy compared with placebo and chemotherapy in patients with primary invasive triple-negative breast cancer. In: ClinicalTrials.gov. U.S. National Library of Medicine. 2017. https ://clinicaltrials.gov/ct2/show/ NCT03197935. Accessed 10 April 2018.

41. Fondazione Michelangelo. Neo-adjuvant study with the pdl1directed antibody in triple negative locally advanced breast cancer undergoing treatment with nab-paclitaxel and carboplatin. In: ClinicalTrials.gov. U.S. National Library of Medicine. 2017. https ://clinicaltrials.gov/ct2/show/ NCT02620280. Accessed 10 April 2018.

42. Nanda R, Liu MC, Yau C, Asare S, Hylton N, Veer V, et al. Pembrolizumab plus standard neoadjuvant therapy for high-risk breast cancer (BC): Results from I-SPY 2. J Clin Oncol. 2017;35:abst 506. 